\title{
Genetic variance modifies apoptosis susceptibility in mature oocytes via alterations in DNA repair capacity and mitochondrial ultrastructure
}

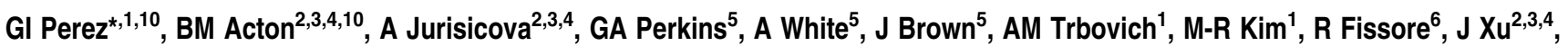 \\ A Ahmady ${ }^{2,3,4}$, SG D'Estaing ${ }^{2,3,4}$, H Li $^{2,3,4}$, W Kagawa ${ }^{7}$, H Kurumizaka ${ }^{7}$, S Yokoyama ${ }^{7}$, H Okada ${ }^{8,9}$, TW Mak $^{8,9}$, MH Ellisman ${ }^{5}$, \\ RF Casper ${ }^{2,3,4}$ and JL Tilly*,1
}

\begin{abstract}
Although the identification of specific genes that regulate apoptosis has been a topic of intense study, little is known of the role that background genetic variance plays in modulating cell death. Using germ cells from inbred mouse strains, we found that apoptosis in mature (metaphase II) oocytes is affected by genetic background through at least two different mechanisms. The first, manifested in AKR/J mice, results in genomic instability. This is reflected by numerous DNA double-strand breaks in freshly isolated oocytes, causing a high apoptosis susceptibility and impaired embryonic development following fertilization. Microinjection of Rad51 reduces DNA damage, suppresses apoptosis and improves embryonic development. The second, manifested in FVB mice, results in dramatic dimorphisms in mitochondrial ultrastructure. This is correlated with cytochrome $c$ release and a high apoptosis susceptibility, the latter of which is suppressed by pyruvate treatment, Smac/DIABLO deficiency, or microinjection of 'normal' mitochondria. Therefore, background genetic variance can profoundly affect apoptosis in female germ cells by disrupting both genomic DNA and mitochondrial integrity.
\end{abstract}

Cell Death and Differentiation (2007) 14, 524-533. doi:10.1038/sj.cdd.4402050; published online 13 October 2006

Eukaryotic cells have developed specific mechanisms for monitoring and responding to environmental and genetic perturbations, including defense responses that culminate in DNA repair and survival or the activation of apoptosis. ${ }^{1-3}$ In many cell types, mitochondria are principal determinants of cell fate, not only by controlling energy production via oxidative phosphorylation but also by virtue of their direct role in the regulation of cell death. A number of studies now support the existence of an intracellular checkpoint that monitors mitochondrial integrity, and coordinates proapoptotic and antiapoptotic signals that converge on or emanate from mitochondria. ${ }^{3}$ This checkpoint and the genomic DNA checkpoint mentioned above represent two of the most important surveillance mechanisms available to cells for maintaining homeostasis.

Constant monitoring of mitochondria, like genomic DNA, is essential to the cell, as these organelles provide energy for the cell in the form of ATP through a process dependent on cytochrome $c$. This latter protein is normally located in the mitochondrial intermembrane space. However, potentially lethal stimuli can trigger cytochrome $c$ release into the cytosol through a process involving outer mitochondrial membrane permeabilization that is tightly regulated by $\mathrm{Bcl}-2$ family members. ${ }^{1,3}$ Once released, cytochrome $c$ has been shown to bind with Apaf-1 and facilitate formation of a multimeric protein complex termed the apoptosome, which then serves as an apical activator of a caspase-driven proteolytic cascade that eventually dismantles the cell for phagocytosis. ${ }^{4}$

In addition to ensuring mitochondrial stability, cells must also maintain DNA integrity to avoid cellular transformation or death. In this regard, eukaryotic cells have developed highly sophisticated responses to DNA double-strand breaks (DDSB), including repair pathways involving homologous recombination driven by the Rad51 gene product. ${ }^{5}$ However, irreparable or excessive DDSB activate the DNA surveillance checkpoint and trigger apoptosis, often through p53-dependent recruitment of core proapoptotic proteins such as Bax that destabilize mitochondria and thus lead to apoptosome formation and the caspase cascade. ${ }^{2,6}$

Although a relatively detailed blueprint of specific genes and pathways involved in signaling for, and executing, apoptosis following disruption of either mitochondrial or genomic

\footnotetext{
${ }^{1}$ Vincent Center for Reproductive Biology, Vincent Obstetrics and Gynecology Service, Massachusetts General Hospital/Harvard Medical School, Boston, MA, USA; ${ }^{2}$ Samuel Lunenfeld Research Institute, Mount Sinai Hospital, Toronto, Ontario, Canada; ${ }^{3}$ Department of Physiology University of Toronto, Toronto, Ontario, Canada; ${ }^{4}$ Department of Obstetrics and Gynecology, University of Toronto, Toronto, Ontario, Canada; ${ }^{5}$ Department of Neurosciences and National Center for Microscopy and Imaging Research, University of California-San Diego, La Jolla, CA, USA; ${ }^{6}$ Department of Veterinary and Animal Sciences, University of Massachusetts, Amherst, MA, USA; ${ }^{7}$ Protein Research Group, RIKEN Genomic Sciences Center, Tsurumi, Yokohama, Japan; ${ }^{8}$ Campbell Family Institute for Breast Cancer Research, University Health Network, University of Toronto, Toronto, Ontario, Canada and ${ }^{9}$ Ontario Cancer Institute, Toronto, Ontario, Canada

*Corresponding authors: JL Tilly, Vincent Center for Reproductive Biology, Massachusetts General Hospital, THR-901B, 55 Fruit Street, Boston, MA 02114, USA.

Tel: + 1617724 2182; Fax: + 1617726 0561; E-mail: jtilly@ partners.org or Gl Perez, Department of Physiology, Michigan State University, East Lansing, Ml 48824, USA. Tel: + 1517355 6475; Fax: +1 517355 5125; E-mail: perezg@msu.edu

${ }^{10}$ These two authors contributed equally to this paper

Keywords: apoptosis; DNA damage; mitochondria; genetic modifiers; germ cells; oocytes

Abbreviations: DDSB, DNA double-strand break(s); EM, electron microscopy(ic); ROS, reactive oxygen species

Received 14.2.06; revised 12.9.06; accepted 12.9.06; Edited by B Zhivotovsky; published online 13.10.06
} 
integrity has been formulated over the past decade or so, ${ }^{1-4}$ essentially nothing is known of the role played by background genetic variance in modulating cell death susceptibility. Here, we show from a comparative study of inbred mouse strains that genetic modifiers of apoptosis susceptibility exist in mammals, which, depending on the strain analyzed, phenotypically present as dramatic defects in DNA or mitochondrial stability. Experimental correction of either defect returns the apoptotic threshold of the cells to a low basal level, confirming a direct causal relationship between each genetic background modifier and the enhancement of cell death.

\section{Results}

Apoptosis susceptibility differs among genetic strains. Mature (metaphase II) oocytes obtained by superovulation of adult female mice undergo apoptosis when maintained in vitro, ${ }^{7-9}$ and a large volume of published work supports that this occurs through a genetic program involving Bcl-2 family members and caspases. ${ }^{10}$ During our studies of apoptosis using female germ cells as a model, we noted considerable variation in the incidence of apoptosis in oocytes collected from different inbred strains of mice. Specifically, oocytes from AKR/J and FVB mice exhibited the highest incidence of apoptosis when compared with the low level of death observed in oocytes of either $\mathrm{C} 57 \mathrm{BL} / 6^{7-9}$ or B6C3F1 (Figure 1) mice. Moreover, the high incidence of apoptosis observed in FVB oocytes cultured in vitro for $24 \mathrm{~h}$ (Figure 1) closely matched that detected in FVB oocytes $24 \mathrm{~h}$ after ovulation in vivo (81 $\pm 9 \%$; mean \pm S.E.M., $n=104$ oocytes).

Faulty DNA damage repair in $A K R / J$ mice enhances apoptosis. To uncover the basis of this genetic straindependent variability in cell death, we first assessed DNA integrity given that DNA damage can activate apoptosis. ${ }^{2}$ Genomic DNA integrity in freshly isolated AKR/J oocytes was compromised, as evidenced by a high degree of pre-existent DDSB (Figure $2 \mathrm{c}$ and $\mathrm{d}$ ). The percent of damaged DNA in $A K R / J$ oocytes remained high (viz. unrepaired) after $6 \mathrm{~h}$ of

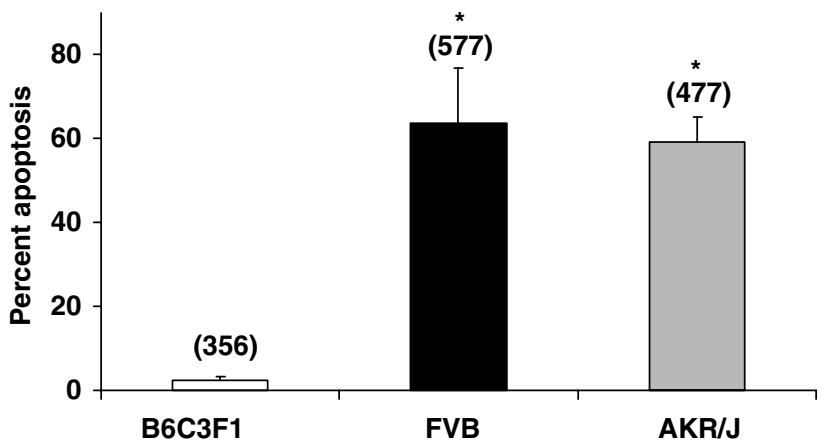

Figure 1 Genetic background alters germline apoptosis susceptibility Percentage of oocytes collected from adult B6C3F1, FVB and AKR/J female mice that exhibited apoptosis after a 24-h culture. Values are the mean ( \pm S.E.M.) of combined data from three independent experiments analyzing the total number of oocytes of each strain indicated over the respective bar (asterisks, $P<0.05$ versus $\mathrm{B} 6 \mathrm{C} 3 \mathrm{~F} 1)$ culture ( $80 \pm 6 \%$; mean \pm S.E.M., $n=49$ oocytes). In contrast, B6C3F1 and FVB oocytes harbored mostly intact DNA, irrespective of whether the analyses were performed using oocytes that were freshly isolated (Figure 2a, b and d)

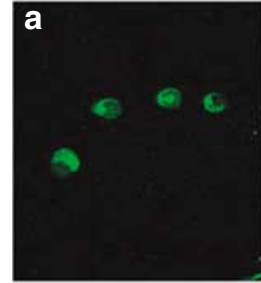

B6C3F1

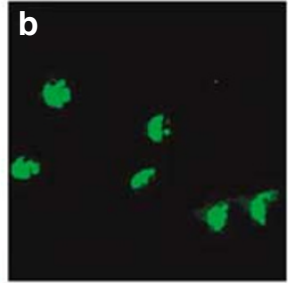

FVB

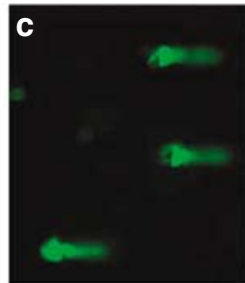

AKR/J d

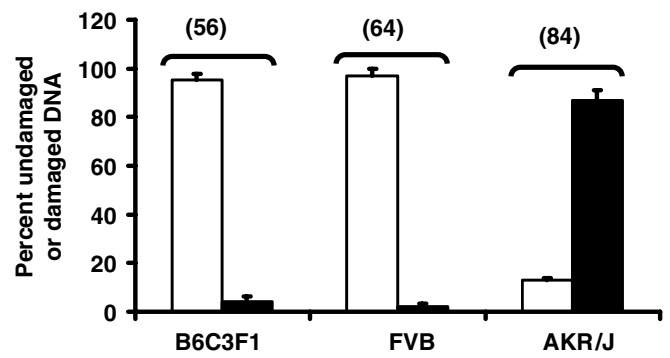

e

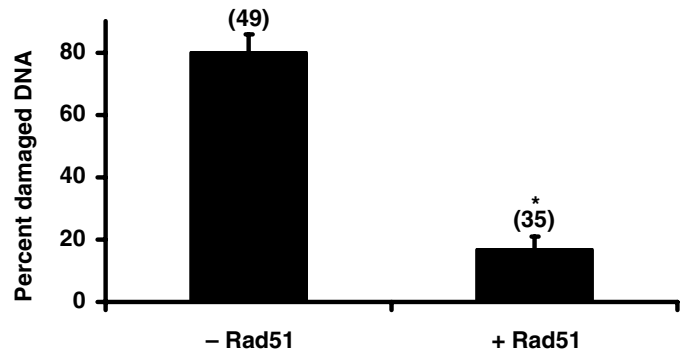

$f$

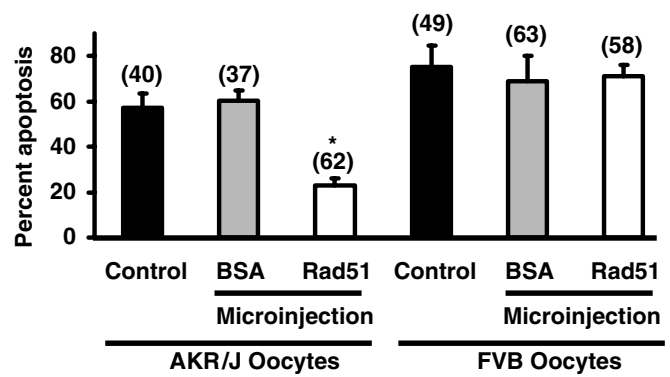

Figure 2 Defective DNA repair enhances apoptosis susceptibility in the AKR/J background. (a-c) Comet assay analysis of DNA integrity in freshly isolated B6C3F1, FVB or AKR/J oocytes. (d) Percent of undamaged (open bars) and damaged (filled bars) DNA in freshly isolated oocytes of the three indicated genetic strains. Values are the mean ( \pm S.E.M.) of combined data from three independent experiments analyzing a total 56-84 oocytes per strain as indicated over each bar. (e) Microinjection of recombinant Rad51 protein ( + Rad51) reduces the percent of damaged DNA in AKR/J oocytes following a 6-h incubation. Values are the mean $( \pm$ S.E.M.) of combined data from three independent experiments analyzing the total number of oocytes indicated per group over each bar (-Rad51, noninjected AKR/J oocytes; asterisk, $P<0.05$ versus noninjected group). (f) Microinjection of recombinant Rad51 suppresses apoptosis in AKR/J oocytes but has no effect on the high incidence of apoptosis in FVB oocytes studied in parallel. Results from analysis of noninjected (Control) and BSA-injected oocytes of each strain are also provided. Values are the mean ( \pm S.E.M.) of combined data from three independent experiments analyzing the total number of oocytes indicated per group over each bar (asterisk, $P<0.05$ versus all other groups) 
or cultured for $6 \mathrm{~h}$ (data not shown). Microinjection of recombinant Rad51 into $A K R / J$ oocytes decreased the extent of DDSB (Figure 2e) and suppressed apoptosis (Figure 2f), confirming that the pre-existent DDSB were causally involved in the high apoptosis susceptibility. In contrast, microinjection of Rad51 had no effect on apoptosis in FVB oocytes (Figure 2f).

Rad51 reverses the reduced developmental competency in AKR/J embryos. Female AKR/J mice have fewer litters and reduced litter sizes compared with other commonly studied mouse strains (http://www.informatics.jax.org/). To determine if defective DNA repair, and the resultant enhancement of apoptosis, observed in the AKR/J germ line contribute to their inferior reproductive performance, the competency of $A K R / J$ oocytes to be fertilized and proceed through preimplantation embryonic development was assessed. In vitro fertilization rates using AKR/J oocytes were significantly lower than those using either B6C3F1 or C57BL/6 oocytes (Figure 3a). Once fertilized, 94\% of the B6C3F1 zygotes and $49 \%$ of the C5LBL/6 zygotes were competent to reach the blastocyst stage. In contrast, less than $10 \%$ of the fertilized AKR/J oocytes were able to complete blastocyst development (Figure $3 b$ ). However, microinjection of Rad51 into fertilized AKR/J oocytes resulted in a pronounced rescue of this defect in that more than $30 \%$ of the microinjected AKR/J zygotes completed development to the blastocyst stage (Figure $3 b$ ). Fertilization rates were not improved when Rad51 was microinjected into mature AKR/J oocytes before fertilization (data not shown), possibly due to Rad51 degradation before sperm penetration as microinjected oocytes need a $2-3 \mathrm{~h}$ recovery period before fertilization.

\footnotetext{
Strain-dependent differences in mitochondrial metabolic function. The absence of DNA damage and the inability of Rad51 microinjection to suppress apoptosis in FVB oocytes suggested that the enhanced death susceptibility observed in this genetic background was unrelated to DNA damage. In light of the prominent role mitochondria play in cell death, ${ }^{3}$ we next analyzed mitochondrial function. Mitochondrial membrane potential in FVB and AKR/J oocytes was slightly elevated when compared with B6C3F1 oocytes (Supplementary Figure S1). An increased level of mitochondrial activity was also detected in $A K R / J$, but not in FVB, oocytes when compared to B6C3F1 oocytes (Supplementary Figure S1). No strain-dependent differences in oocyte ATP content or glutathione content (baseline, following oxidative insult, or after recovery following oxidative insult) were noted (Supplementary Figure S1). However, the levels of reactive oxygen species (ROS) in freshly isolated FVB oocytes were less than $20 \%$ of those found in B6C3F 1 or $A K R / J$ oocytes (Figure 4a), suggesting that mitochondrial metabolic function was impaired in the FVB germ line. As oocytes favor pyruvate as a substrate for oxidation, we explored the effects of pyruvate on oocyte metabolic performance and survival. Treatment of FVB oocytes with pyruvate more than doubled ROS content (Figure 4b) and completely suppressed apoptosis (Figure 4c).
}
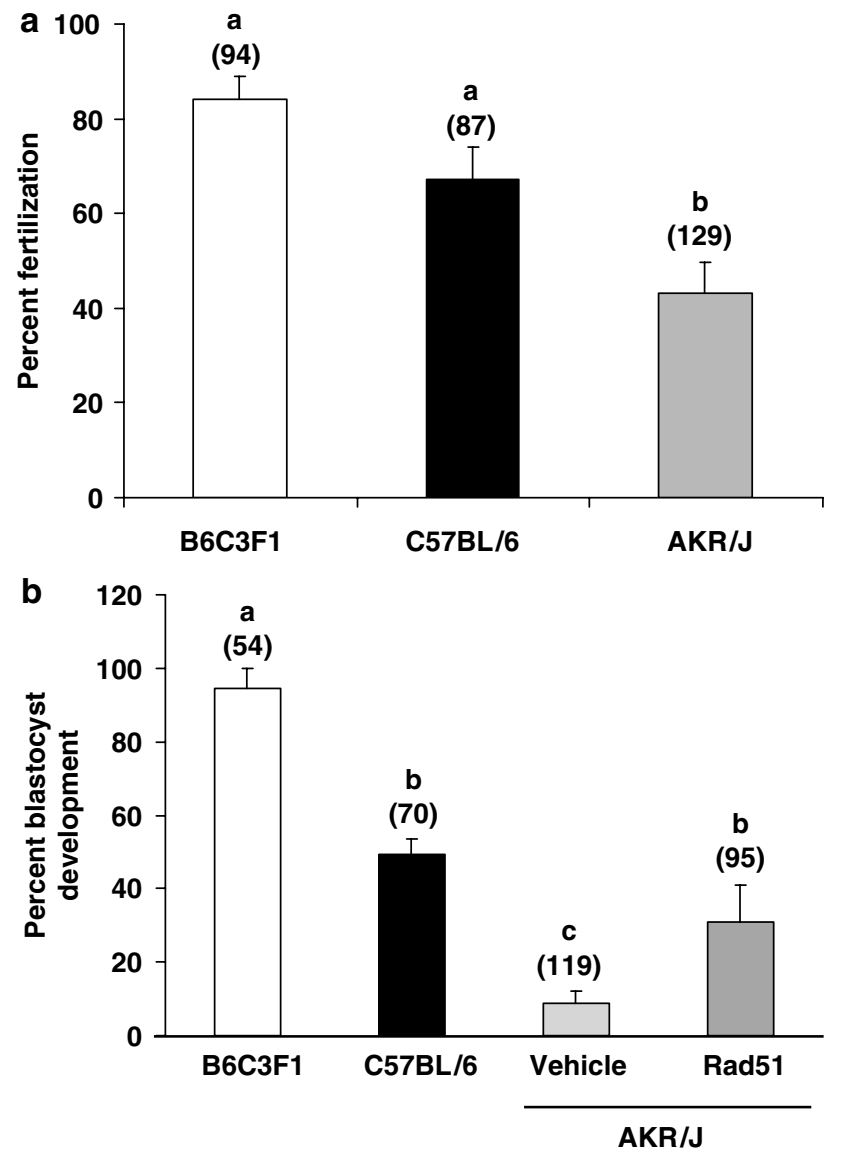

Figure 3 Rad51 rescues the compromised developmental competence of female AKR/J germ cells. (a) In vitro fertilization rates of B6C3F1, C57BL/6 and AKR/J oocytes. Values are the mean ( \pm S.E.M.) of combined data from three independent experiments analyzing the total number of oocytes indicated per group over each bar (different letters, $P<0.05$ ). (b) Pre-implantation embryonic developmental competence of B6C3F1, C57BL/6 and AKR/J zygotes. Microinjection of recombinant Rad51 protein into $A K R / J$ zygotes increases blastocyst formation rates to levels not different from those observed with C57BL/6 zygotes. Values are the mean $( \pm$ S.E.M.) of combined data from three independent experiments analyzing the total number of zygotes indicated per group over each bar (different letters, $P<0.05$ )

Mitochondrial microinjection reduces the high apoptosis susceptibility in FVB oocytes. To test if mitochondrial dysfunction in FVB oocytes directly contributes to their high apoptosis susceptibility, mitochondria collected from FVB mice were microinjected into B6C3F1 oocytes, and the incidence of apoptosis over a subsequent 24-h culture period was recorded. Microinjection of vehicle did not affect the low basal rate of apoptosis seen in oocytes of this strain; however, microinjection of FVB mitochondria into B6C3F1 oocytes significantly increased apoptosis when compared with those levels observed in noninjected or vehicle-injected controls (Figure 5a) Conversely, microinjection of mitochondria collected from B6C3F1 mice into FVB oocytes reduced apoptosis by approximately $50 \%$ compared with noninjected or vehicle-injected controls (Figure 5b). Microinjection of mitochondria derived from mouse embryonic stem cells, which possess mitochondria that are more similar 


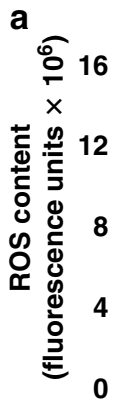

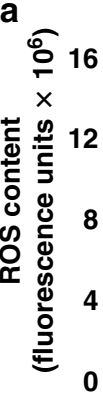
(53)

b

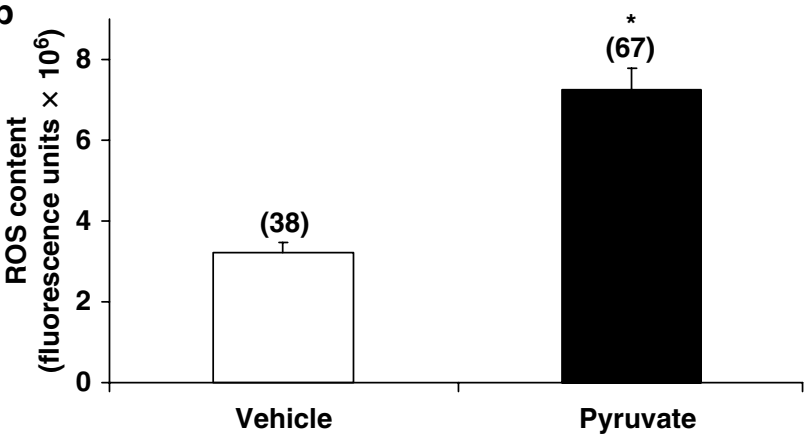

C

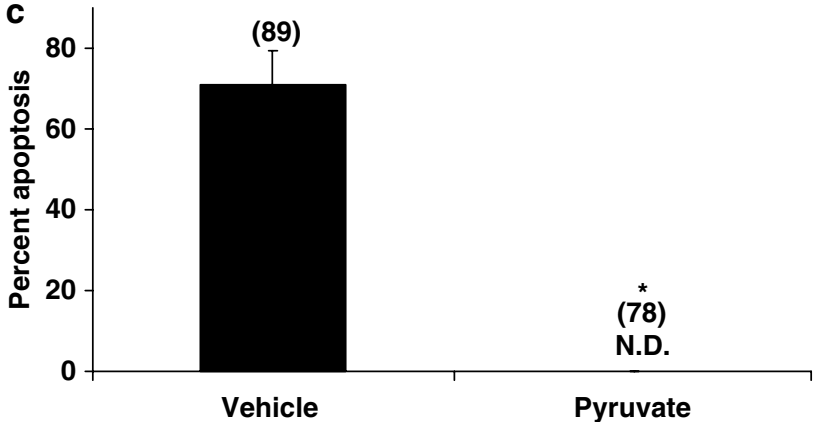

Figure 4 Pyruvate reverses impaired ROS output and prevents apoptosis in FVB germ cells. (a) Freshly isolated FVB oocytes possess extremely low levels of ROS compared to oocytes of the other strains, indicative of reduced mitochondrial metabolic function. Values are the mean $( \pm$ S.E.M.) of combined data from three independent experiments analyzing the total number of oocytes of each strain as indicated over the respective bar (asterisk, $P<0.05$ versus $\mathrm{B} 6 \mathrm{C} 3 \mathrm{~F} 1$ or $\mathrm{AKR} / \mathrm{J}$ ). (b) Incubation of FVB oocytes in the presence of $10 \mathrm{mM}$ pyruvate for $6 \mathrm{~h}$ increases ROS content to levels approaching those observed in freshly isolated B6C3F1 oocytes (see Figure $4 a)$. Values are the mean $( \pm$ S.E.M.) of combined data from three independent experiments analyzing the total number of oocytes indicated per group over each bar. (c) Pyruvate completely reverses the high apoptosis susceptibility observed in vehicle-exposed FVB oocytes cultured for $24 \mathrm{~h}$ in parallel. Values are the mean $( \pm$ S.E.M.) of combined data from three independent experiments analyzing the total number of oocytes indicated per group over each bar (N.D., none detected)

to those found in germ cells, also suppressed apoptosis in FVB oocytes (Supplementary Figure S2).

Electron microscopic (EM) tomography reveals structural anomalies in FVB mitochondria. To gain additional insight into the properties of FVB mitochondria that may be involved in elevating apoptosis susceptibility, the
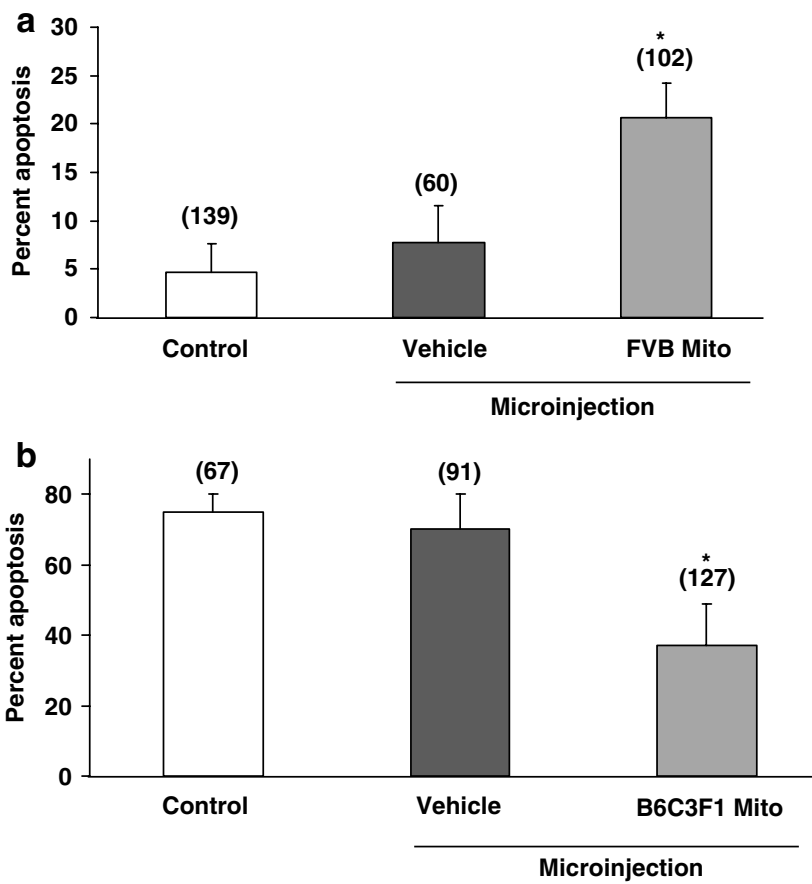

Figure 5 Mitochondrial 'swapping' alters apoptosis susceptibility. Microinjection of approximately $5 \times 10^{3} \mathrm{FVB}$ mitochondria (Mito) into B6C3F1 oocytes increases apoptosis over that observed in noninjected (Control) or vehicle-injected B6C3F1 oocytes during a subsequent $24 \mathrm{~h}$ culture (a), whereas microinjection of approximately $5 \times 10^{3}$ B6C3F1 mitochondria into FVB oocytes decreases apoptosis over that observed in noninjected (Control) or vehicle-injected FVB oocytes during a subsequent $24 \mathrm{~h}$ culture (b). Values are the mean $( \pm$ S.E.M.) of combined data from three independent experiments analyzing the total number of oocytes indicated per group over each bar (asterisk, $P<0.05$ versus Control or Vehicle)

three-dimensional architecture of individual mitochondria in FVB oocytes was reconstructed using EM tomography and compared with that of B6C3F1 oocyte mitochondria. Mitochondria were found distributed in small clusters in both B6C3F1 (Figure 6a) and FVB (Figure 7a) oocytes. Contacts between mitochondria and the endoplasmic reticulum were often observed in oocytes of both strains (Figures 6a, 7a and b). Despite these similarities, striking differences in ultrastructural features of mitochondria in B6C3F1 and FVB oocytes were uncovered. Mitochondria in B6C3F1 oocytes typically displayed a dichotomy of cristae structure, in which one-half appeared as a single large crista. The other half consisted of one to four lamellar cristae, with one lamellar crista consistently located around the periphery of the mitochondrion (Figure 6a-e; Supplementary Video S1). Crista junctions were commonly observed in these mitochondria as well.

By comparison, mitochondria in freshly isolated FVB oocytes frequently exhibited a number of degenerative features not observed in mitochondria of freshly isolated B6C3F1 oocytes. For example, the large crista that was so prominent in mitochondria of B6C3F1 oocytes (Figure 6) was absent in many of the mitochondria in FVB oocytes (Figure 7a and b; Supplementary Video S2). In its place was found a centrally located matrix. Another prominent difference was 


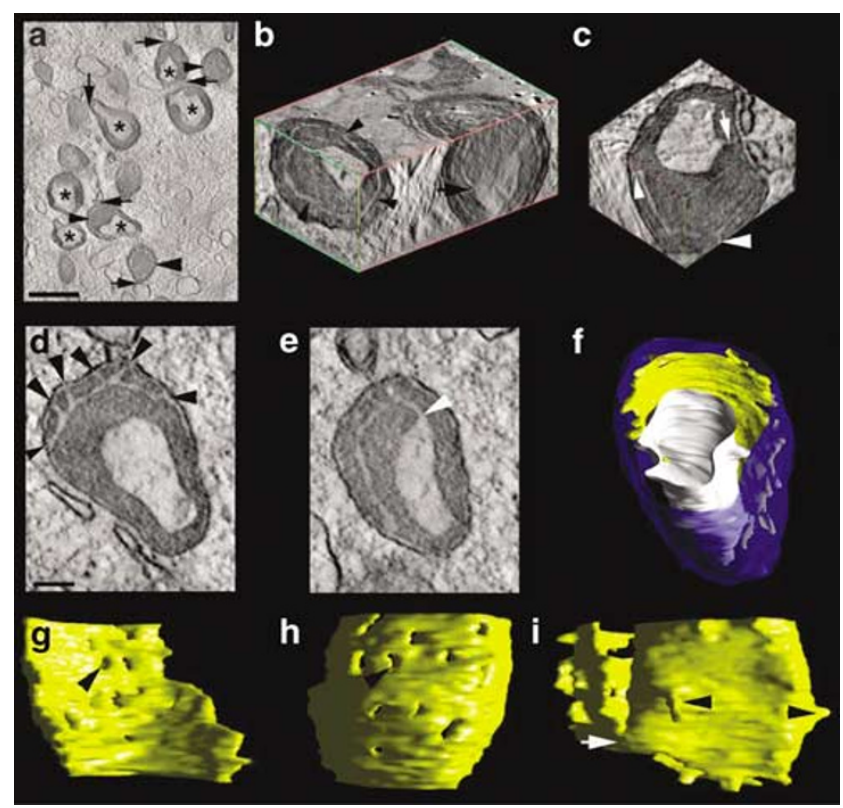

Figure 6 Electron microscopy-based tomographic reconstructions of $\mathrm{B} 6 \mathrm{C} 3 \mathrm{~F} 1$ oocyte mitochondria (see also Supplementary Video S1). (a) A $1.1 \mathrm{~nm}$ slice from a three-dimensional reconstruction of a volume of a B6C3F1 oocyte with a cluster of mitochondria visible. The mitochondrial matrices are dark, indicating an absence of matrix swelling. The mitochondria typically display a dichotomy of cristae structure. One-half of the dichotomy is a single, large, vacuolated cristal compartment (asterisks), whereas the other half consists of one to four lamellar compartments. One of these lamellar cristae is always observed around the periphery of the mitochondrion (arrowheads). Contacts between mitochondria and the endoplasmic reticulum are often observed (arrows). Scale bar represents $500 \mathrm{~nm}$. (b) Smaller cube through the volume showing three perpendicular faces through the interior of two of the mitochondria shown in Figure 6a. The lamellar cristae are often observed as arches or shells that extend around a large portion of the periphery (arrowheads). The vacuolated crista connects to a peripheral crista via a more tubular junction (arrow). (c) Smaller cube through the volume showing three perpendicular faces through the interior of another mitochondria shown in Figure 6a that was volume segmented. All three faces show examples of crista junctions. Two of the crista junctions connect the peripheral crista to the intermembrane space (arrowheads). The other crista junction connects the vacuolated crista to the same space on the other side of the mitochondrion (arrow). (d) A $1.1 \mathrm{~nm}$ slice through the mitochondrion shown in Figure 6c. It emphasizes a typical example of many crista junctions connecting one broad side of the peripheral crista to the intermembrane space. There are six crista junctions seen in this thin slice (arrowheads). Scale bar represents $100 \mathrm{~nm}$. (e) Another thin slice through the same reconstruction shown in Figure $6 \mathrm{~d}$. Note how the large vacuolated crista connects via a tubular opening (arrowhead) to the peripheral crista, making them essentially one crista, albeit with compartments of different architecture. Because of the interconnectedness, this mitochondrion possessed only the one crista. Scale is the same as that shown in Figure $6 \mathrm{~d}$. (f) The mitochondrion in Figure $6 \mathrm{c}-\mathrm{e}$ was segmented along its membranes. Even though contiguous, the large, vacuolated cristal compartment (white) was segmented separately from the lamellar cristal compartment (yellow) to aid in the analysis. The outer membrane is shown in blue and made translucent to better visualize the crista. $(\mathbf{g}, \mathbf{h})$ Two views looking from the inside and outside, respectively, of the matrix portion, between the intermembrane space and the peripheral cristal compartment. The matrix is curved and has a fenestrated appearance because of the crista junctions. There are 11 crista junctions; for reference, the arrowhead in each panel indicates the same junction. (i) $A$ single view of the peripheral crista on the left and the vacuolated crista in the center and right. These structures are joined by a tubular connection at only one end (arrow). The vacuolated portion of the crista has fewer crista junctions, although about the same surface area as the lamellar portion of the crista. Two of the few crista junctions for the vacuolated crista are indicated (arrowheads) that the peripheral cristae in mitochondria of FVB oocytes had transformed into 'onion-like' whorls (Figure $7 \mathrm{~b}$ and $\mathrm{c}$ ). Moreover, crista junctions were no longer discernable, and it was common to find ruptured outer mitochondrial membranes that allowed the inner boundary membranes to extend outward (Figure 7a-g). Other mitochondria in FVB oocytes possessed vacuolated cristae, but these cristae were adorned with abnormal internal 'blobs', possibly representing orphaned satellite volumes of the matrix (Figure 7a).

As pyruvate was found to inhibit apoptosis in FVB oocytes (Figure 4c), we next explored if pyruvate exerted its effects at the level of mitochondrial ultrastructure. In FVB oocytes exposed for $3 \mathrm{~h}$ to pyruvate, the majority of mitochondria exhibited features similar to those of mitochondria in $\mathrm{B} 6 \mathrm{C} 3 \mathrm{~F} 1$ oocytes (Figure $7 \mathrm{~h}-\mathrm{k}$; Supplementary Video S3). The large crista, and both the transverse and peripheral cristae, were present in the same dichotomy, and crista junctions were prominent (Figure $7 \mathrm{~h}-\mathrm{j}$ ). Scoring of mitochondria with abnormal morphology in random sections of $\mathrm{B} 6 \mathrm{C} 3 \mathrm{~F} 1$ oocytes, as well as in untreated and pyruvate-treated FVB oocytes, revealed that pyruvate reduced the number of abnormal mitochondria in FVB oocytes by more than $75 \%$ compared with vehicle-treated FVB oocytes (Figure 7k).

Ultrastructural anomalies in FVB mitochondria facilitate cytochrome $c$ release. The presence of large perforations in the outer membranes of mitochondria in freshly isolated FVB oocytes (Figure 7c-g), and the ability of pyruvate to reduce both the number of abnormal mitochondria (Figure 7k) and the onset of apoptosis (Figure 4c) in FVB oocytes, suggested that the ultrastructural anomalies in mitochondria of oocytes from this strain directly contribute to their high apoptosis susceptibility. In keeping with this, and past studies showing that mitochondrial cytochrome $c$ release plays a central role in the execution of apoptosis, ${ }^{4}$ we found that during short-term incubations, mitochondria collected from FVB oocytes released 19\% more cytochrome $c(56 \pm 15 \%$ of the total mitochondrial cytochrome $c$ pool present before incubation was released; mean \pm S.E.M., $n=4$ with 300 oocytes/experiment) than did mitochondria from $\mathrm{B} 6 \mathrm{C} 3 \mathrm{~F} 1$ oocytes $(37 \pm 8 \%$ released; mean \pm S.E.M., $n=4$ with 300 oocytes/experiment). However, when mitochondria were isolated from FVB oocytes preincubated for $2 \mathrm{~h}$ with pyruvate, there was a reduction in the amount of cytochrome $c$ released during the subsequent incubation period $(34 \pm 2 \%$ released; mean \pm S.E.M., $n=4$ with 200 oocytes/experiment) to levels comparable to those released by $\mathrm{B} 6 \mathrm{C} 3 \mathrm{~F} 1$ mitochondria.

Cytochrome $c$ and Smac/DIABLO synergize to promote apoptosis. Given this, we then determined if cytochrome $c$, when experimentally elevated in the cytoplasm of oocytes, could directly activate apoptosis. Microinjection of cytochrome $c$ into B6C3F1 oocytes, which have a low basal rate of apoptosis (Figure 1), did not affect the incidence of apoptosis in a subsequent 24-h culture (Figure 8a). However, microinjection of recombinant Smac/DIABLO, a cell death-promoting protein released along with cytochrome $c$ from mitochondria, ${ }^{11,12}$ increased apoptosis in B6C3F1 oocytes by almost three-fold. Microinjection of both 


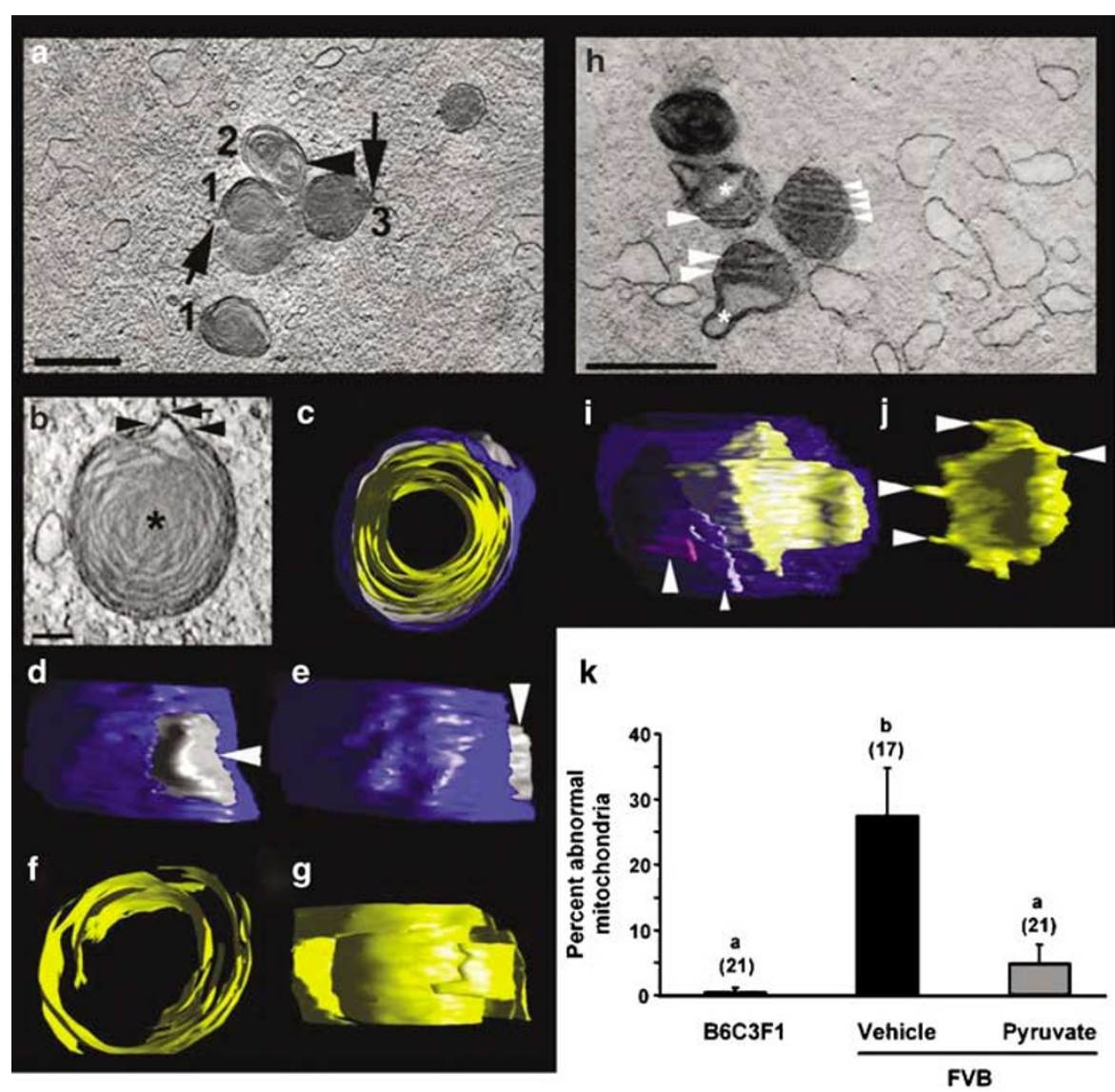

Figure 7 Electron microscopy-based tomographic reconstructions of FVB oocyte mitochondria (see also Supplementary Videos S2 and S3). (a) A $1.1 \mathrm{~nm}$ slice from a three-dimensional reconstruction of a volume of a FVB oocyte showing mitochondria with a spectrum of ultrastructural anomalies. The two mitochondria labeled '1' are the types most commonly seen. The vacuolated cristal compartment prevalent in B6C3F1 mitochondria (see Figure 6) is noticeably missing. The matrix occupies the central volume; however, the cristae appear to have degraded to 'onion-like' whorls seen around the periphery. The mitochondrion labeled '2' has a much-reduced matrix and in its place whorls of membranes are found. Its periphery also has whorls similar to those of the mitochondria labeled '1'. However, this mitochondrion does have a vacuolated crista (arrowhead) that nevertheless is abnormal in that it possesses internal 'blobs' that may be orphaned matrix subvolumes. The mitochondrion labeled ' 3 ' appears more like that in B6C3F1 0ocytes, with the exception that it too lacks the large vacuolated cristal compartment. There is evidence of limited, localized 'whorling' membrane degradation; however, most of the lamellar cristal compartment appears nearly normal. Arrows note contacts between mitochondria and vesicles, which may be derived from the endoplasmic reticulum. Scale bar represents $500 \mathrm{~nm}$. (b) A $1.1 \mathrm{~nm}$ slice through the lower volume of mitochondrion ' 1 ' shown in Figure $7 \mathrm{a}$. The central matrix compartment (asterisk) is surrounded by whorls of membranes likely from degraded cristae. The outer membrane is ruptured (arrowheads) allowing the inner boundary membrane to extend outward (arrow). Scale bar represents $100 \mathrm{~nm}$. (c) The segmented and surface-rendered mitochondrion shown in Figure $7 \mathrm{~b}$. The outer membrane is shown in blue, the inner boundary membrane in gray/white, and the membrane whorls in yellow. This segmented volume shows that the whorls extend throughout the volume and are almost concentric in nature. (d, e) Two side views of the surface-rendered volume from Figure $7 \mathrm{c}$ that emphasize the extent of outer membrane tearing (arrowhead in Figure $7 \mathrm{~d}$ ) and blowout of the inner boundary membrane (arrowhead in Figure 7e). (f, g) Two perpendicular views (top and side) of the subset of membrane whorls in Figure 7c that are broken. (h) A $1.1 \mathrm{~nm}$ slice from a three-dimensional reconstruction of a volume of a pyruvate-treated FVB oocyte showing mitochondria with similar architecture to those in B6C3F1 oocytes (see Figure 6). The large vacuolated cristal compartment (asterisk) and transverse and peripheral cristae (arrowheads) are present in the same dichotomy. Scale bar represents $500 \mathrm{~nm}$. (i) The segmented and surface-rendered mitochondrion shown at the bottom in Figure $7 \mathrm{~h}$. The outer membrane is shown in blue and made translucent to better visualize the cristae, the vacuolated crista in yellow and the two lamellar cristae (arrowheads) in magenta and cyan. The transverse cristae extend less than half-way through the depth of the mitochondrion. (j) The large vacuolated crista of the mitochondrion in Figure $7 \mathrm{~h}$ as seen from the other side. This view emphasizes the four crista junctions (arrowheads) that connect this crista to the intermembrane space. (k) Pyruvate reverses the occurrence of abnormal mitochondrial ultrastructures in FVB oocytes. Values are the mean ( \pm S.E.M.) of combined transmission EM data from analyzing 10-12 random sections of oocytes in each group (the total number of oocytes analyzed is indicated over each bar; different letters, $P<0.05)$

cytochrome $c$ and Smac/DIABLO further increased the incidence of apoptosis in B6C3F1 oocytes over that obtained using Smac/DIABLO alone (Figure 8a). To directly test the in-vivo significance of Smac/DIABLO to FVB oocyte death, mutant mice lacking Smac/DIABLO ${ }^{13}$ were outcrossed onto a FVB genetic background and oocytes were collected for analysis. In keeping with the data obtained from the microinjection studies, the incidence of apoptosis in Smac/DIABLO-deficient FVB oocytes after $24 \mathrm{~h}$ of culture was one-half of that observed in oocytes collected from wildtype sisters cultured in parallel (Figure 8b).

\section{Discussion}

Evidence demonstrating phenotypic variations in traits that are inherited in a simple Mendelian fashion has been available for nearly a century. ${ }^{14}$ Although there are a number of reasons why the simple inheritance of a trait can be phenotypically 

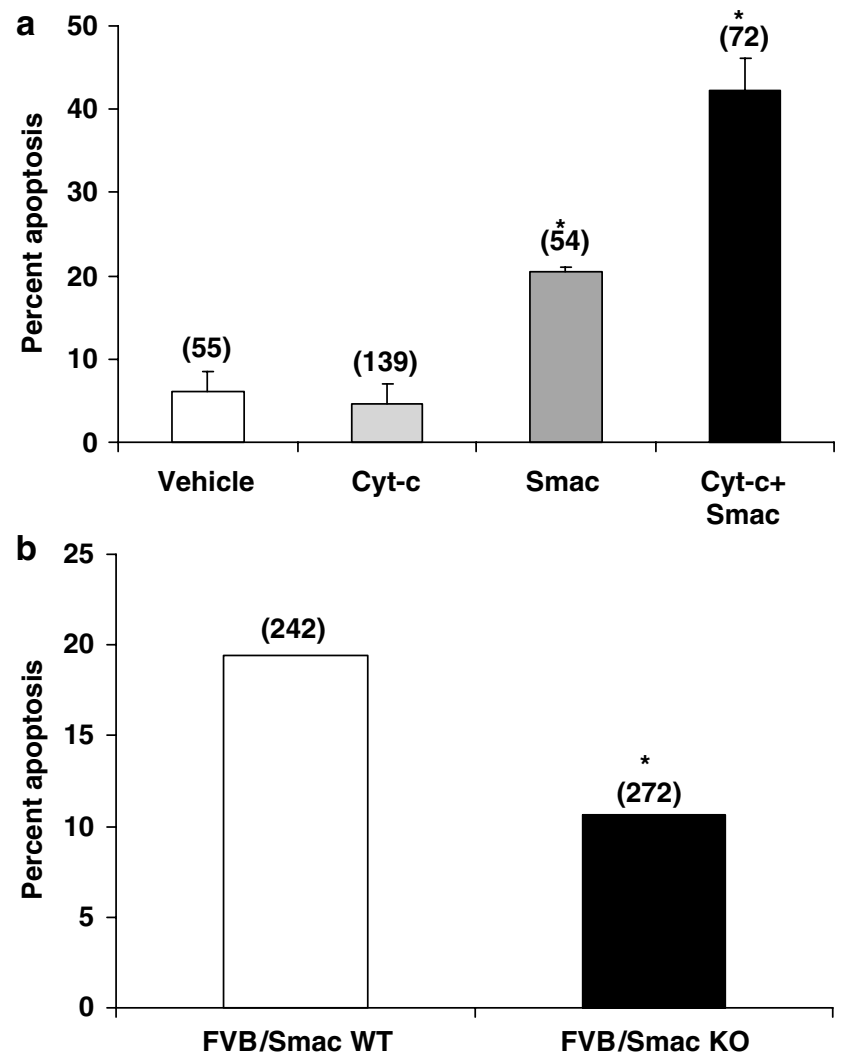

Figure 8 Cytochrome $c$ synergizes with Smac/DIABLO to promote germ cell apoptosis. (a) Effect of microinjecting vehicle, cytochrome $c$ (Cyt-c), recombinant Smac/DIABLO (Smac) or cytochrome $c$ and Smac/DIABLO on the incidence of apoptosis in B6C3F1 oocytes following a 24-h culture. Values are the mean $( \pm$ S.E.M.) of combined data from three independent experiments analyzing the total number of oocytes indicated over each bar (different letters, $P<0.05$ ). (b) Incidence of apoptosis in oocytes collected from F5 generation FVB mice expressing (Smac/DIABLO wild type or Smac WT; $n=14$ mice) or lacking (Smac/ DIABLO knockout or Smac KO; $n=13$ mice) functional Smac/DIABLO. Values are the mean $( \pm$ S.E.M.) of combined data from three independent experiments analyzing the total number of oocytes indicated over each bar (asterisk, $P<0.05$ by $\chi^{2}$ analysis)

variable, one of the most prominent is represented by genetic modifiers. ${ }^{15}$ In humans, the role of modifier genes in disease predisposition has gained considerable attention in recent years as modifier loci that affect development of an array of health problems, including congenital malformations, cystic fibrosis, deafness and cancer, have been reported. The majority of work on genetic modifiers in mammals has, however, originated from studies of inbred mouse strains. ${ }^{15}$ Although in most cases the genes responsible have not yet been determined, the impact of genetic background on the emergence or repression of a given phenotype in mice especially those harboring targeted gene disruptions or ectopically expressed transgenes - has been known for years. In the context of cell death regulation, one of the more obvious examples of this comes from studies of mutant mice lacking the proapoptotic executioner, caspase-3. When originally described, caspase-3 deficiency was reported to result in perinatal lethality due to excessive neural precursor cell expansion and exencephaly during development. ${ }^{16}$
However, after backcrossing caspase-3 deficient mice onto a congenic C57BL/6 background, the brain phenotype was minimized and the perinatal lethality was lost, allowing the mutant animals to survive as adults. ${ }^{17}$

Herein we have uncovered two different mechanisms by which genetic strain modifies apoptosis susceptibility in mammalian oocytes. The first of these, observed in AKR/J mice, manifested as an inability to carry out repair of DDSB. This conclusion is based on the finding that a high degree of pre-existent DDSB is present in freshly isolated AKR/J germ cells. This phenotype was minimized by microinjection of Rad51, which was followed by a marked reduction in apoptosis. The reduced DNA repair capacity seen in AKR/J germ cells was also associated with a severe impairment in embryonic development following fertilization, which likely explains the poor reproductive outcomes previously reported for AKR/J mice (http://www.informatics.jax.org/). Of additional note, the life expectancy of AKR/J mice is 10 months or less under conventional housing conditions (unpublished findings; http://www.informatics.jax.org/). This is much shorter than that of many other mouse strains, including FVB, C57BL/6 and $\mathrm{B} 6 \mathrm{C} 3 \mathrm{~F} 1$, which routinely live past 24 months (unpublished findings; http://www.informatics.jax.org/). AKR/J mice also exhibit a high predisposition for leukemia (http:// www.informatics.jax.org/). Although we do not know if the cancer predisposition or early death of $A K R / J$ mice is related to the molecular phenotype identified herein, such an outcome would be in keeping with past studies linking DNA instability and defective DNA repair to both cancer and aging. ${ }^{18,19}$

The second modifier of apoptosis manifested as a striking mitochondrial defect in oocytes of FVB mice, which is of particular interest in that transgenic mice are most frequently generated on this genetic background. Accordingly, phenotypes of transgenic lines produced using FVB zygotes for pronuclear injection - especially those involving the ectopic expression of apoptosis-regulatory genes - may reflect the outcome of a much more complex genetic interplay than simply the impact of the transgene. In any case, when compared with B6C3F1 or AKR/J oocytes, FVB oocytes showed a marked reduction in ROS content, indicative of diminished metabolic activity. Further to this, analysis of mitochondrial ultrastructure indicated a myriad of abnormalities in mitochondrial membrane composition and cristae in FVB oocytes. The most striking of these were the onion-like whorling of the peripheral cristae and the frequent sites of outer mitochondrial membrane rupture. A similar pathophysiology has been observed in heart cells exposed to stress, ${ }^{20}$ and in yeast whose energy production is impaired, ${ }^{21}$ indicating that this phenotype is not unique to the germ line of FVB mice. Moreover, the loss of cristae in mitochondria of yeast maintained under anoxic conditions is reversed by aeration, ${ }^{22}$ suggesting that tremendous plasticity exists in mitochondrial ultrastructure and function. In agreement with this, both the structural anomalies in mitochondria and the deficiency in ROS production were rapidly reversed when FVB oocytes were cultured with pyruvate. These outcomes were paralleled by a complete absence of apoptosis in pyruvate-treated FVB oocytes, highlighting a direct causal relationship between these mitochondrial defects and the high susceptibility to apoptosis. 
This conclusion is further supported by the finding that microinjection of FVB mitochondria into B6C3F1 oocytes, which have an inherently low basal rate of death, increased the incidence of apoptosis in these cells by nearly five-fold. We interpreted these results to indicate that FVB mitochondria are supplying proapoptotic factors to the B6C3F1 oocytes, most likely by virtue of their 'leaky' outer membrane structure. Consistent with this, mitochondria collected from FVB oocytes released approximately $20 \%$ more cytochrome $c$ in short-term incubations than did mitochondria of B6C3F1 oocytes. However, direct microinjection of cytochrome $c$ into B6C3F1 oocytes did not, by itself, induce apoptosis, suggesting that the FVB mitochondria must be supplying additional proapoptotic factors. To this end, microinjection of recombinant Smac/ DIABLO increased apoptosis in B6C3F1 oocytes, and coinjecting cytochrome $c$ synergistically enhanced this effect. Although Smac/DIABLO-deficient mice were reported to have no phenotype, ${ }^{13}$ outcrossing these mutants onto a FVB background has now uncovered a central role for this gene product in executing apoptosis in the germ line of animals with disrupted mitochondrial integrity.

In summary, we have identified mechanisms through which genetic modifiers exert profound negative effects on DNA or mitochondria stability in female germ cells, both of which underlie a marked elevation in apoptosis susceptibility. It remains unknown when and how these phenotypes emerge during the formation and maturation of the female germ line, but a comparable characterization of less mature oocyte stages is ongoing in our laboratory to address these questions. Further, while the specific genes responsible for conveying these effects are yet to be fully characterized, intercrosses of FVB mice with C57BL/6 mice have revealed that the FVB-associated modifier is likely encoded by a single locus, is recessive and is not sex-linked. Additionally, the phenotype in FVB oocytes is rescued by fertilization through a mechanism that may involve the initiation of calcium waves that are triggered by sperm entry (Supplementary Figure S3). In contrast, the AKR/J phenotype is not rescued by fertilization. Although the reason for this is currently unknown, the excessive DDSB and the ensuing high apoptosis susceptibility do not appear tied to strain-dependent alterations in levels or intracellular localization of Rad51 (Supplementary Figure S4). Studies are in progress to narrow down the chromosomal regions in FVB and $A K R / J$ mice that facilitate DNA and mitochondrial instability in oocytes.

\section{Materials and Methods}

Animals. Wild-type AKR/J (Jackson Laboratory, Bar Harbor, ME, USA), FVB (Taconic, Germantown, NY, USA), C57BL/6 (Charles River Laboratory, Wilmington, MA, USA) and B6C3F1 (Charles River Laboratory, Wilmington, MA, USA or SaintConstant, Quebec, Canada) mice between 2 and 4 months of age were purchased for this study. In some experiments, mutant mice lacking Smac/DIABLO ${ }^{13}$ were outcrossed five generations onto a FVB genetic background for analysis. All experiments involving animals described herein were reviewed and approved by the institutional animal care and use committees of Massachusetts General Hospital, Michigan State University, and Mount Sinai Hospital.

Oocyte isolation and culture. Oocytes were collected after superovulation as described. ${ }^{7-9}$ Briefly, ovulated oocytes were denuded of cumulus cells by a 1-min incubation in $80 \mathrm{IU} / \mathrm{ml}$ of hyaluronidase (Sigma, St. Louis, MO, USA), followed by three washes with culture medium. All cultures were carried out in human tubal fluid
(Irvine Scientific, Santa Ana, CA, USA) supplemented with $0.5 \%$ BSA. The oocytes were maintained in $0.1 \mathrm{ml}$ drops of culture medium under paraffin oil, and incubated at $37^{\circ} \mathrm{C}$ in a humidified atmosphere of $5 \% \mathrm{CO}_{2}$ and $95 \%$ air.

In vitro fertilization and embryo culture. Female mice were superovulated as described above and cumulus-oocyte complexes from the indicated strains were mixed with capacitated sperm collected from adult male mice of the respective strain for in vitro fertilization in HTF medium supplemented with $0.5 \% \mathrm{BSA} .{ }^{9}$ After $2 \mathrm{~h}$, the cumulus-0ocyte complexes were washed and maintained in KSOM medium (Specialty Media, Phillipsburg, NJ, USA) to determine fertilization rates (two-cell stage) and monitor embryonic progression. As zygotes progress to the blastocyst stage of development within $96 \mathrm{~h}$ and begin hatching, embryos were fixed at this time and stained with the DNA-binding dye Hoechst 33342 (Sigma) for light and fluorescence microscopic analysis of blastocyst cell number and quality.

Apoptosis analyses. Oocytes were evaluated as described ${ }^{7-9,23}$ for characteristics of apoptosis, including morphological changes (e.g., cellular condensation, budding and fragmentation) and biochemical alterations (i.e., DNA cleavage using Comet Assay Kit; Trevigen, Gaithersburg, MD, USA). To primarily detect DDSB, the comet assay was performed under neutral conditions. ${ }^{24}$ Analysis of DNA 'comets' to generate quantitative data on the percent of undamaged versus damaged DNA was performed using the VisComet program ${ }^{23}$ (Impuls Computergestutzte Bildanalyse GmbH, Gilching, Germany). Positive controls (oocytes exposed to doxorubicin for $24 \mathrm{~h}$, which have a known percentage of damaged DNA: $>80 \%$ ) and negative controls (freshly isolated B6C3F1 oocytes, which have intact DNA) were always run in parallel. To be scored as apoptotic, oocytes had to exhibit these two parameters (cellular fragmentation by morphology and DNA cleavage by the comet assay) simultaneously. The reliability of this approach for the identification of apoptosis in oocytes has been confirmed in past studies with a number of other assays, including activation of caspase-3-like enzymes. $^{8}$

Assessment of MMP. Oocytes were stained using the membrane-sensitive dye JC-1 (DePsipher ${ }^{\mathrm{TM}}$; R\&D Systems, Minneapolis, MN, USA) and then viewed by deconvolution microscopy (Olympus IX70) with fluorescein isothiocyanate (FITC) and rhodamine isothiocyanate (RITC) filters. ${ }^{25}$ For each oocyte, 10 optically sectioned ( $2 \mu \mathrm{m}$ thick) images were captured and analyzed using DeltaVision Software (Applied Precision, LLC., Issaquah, WA, USA) to quantitate fluorescence signal intensity. After subtraction of background noise, the ratio of RITC (Jaggregate) to FITC (J-monomer) was determined for each section, and an average ratio of J-aggregate to J-monomer for the entire oocyte was calculated.

Measurement of ATP. The ATP Bioluminescent Somatic Cell Assay Kit (Sigma, Oakville, Ontario, Canada) was used to assess ATP content in groups of 25 oocytes following extrapolation from a standard curve composed of 10 ATP concentrations ranging between $18 \mathrm{pmol}$ and $7.2 \mu \mathrm{mol}$ per sample volume. Duplicate luminometer readings were taken from each sample over $20 \mathrm{~s}$ intervals, and the average relative light unit readings were used to determine ATP content in the samples against the standard curve.

Bioreduction assay. Oocyte reduction potential was assessed by use of 3(4,5-dimethylthiazol-2-yl)-2,5-diphenyl-tetrazolium bromide (MTT), a water-soluble tetrazolium salt that precipitates as a colored formazan upon reduction. ${ }^{26}$ Groups of 25 oocytes were cultured for $4 \mathrm{~h}$ in $1.2 \mathrm{mM} \mathrm{MTT}$ and then washed several times in phenol-free RPMI medium 1640 (Sigma) supplemented with 0.5\% BSA. The oocytes were then transferred into $100 \mu \mathrm{l}$ of dimethylsulfoxide (DMSO, Sigma) in 96-well plates, and the intensity of the precipitated formazan product was assessed using a plate reader and KC Junior Software (Bio-Tek Instruments, Winooski, VT, USA).

Reduced glutathione (GSH) content. Quantitation of cellular GSH was assayed by the use of monochlorobimane (Molecular Probes, Eugene, OR, USA), a cell-permeant dye that becomes fluorescent upon conjugation to thiol groups. ${ }^{27}$ One group of untreated oocytes served as a control for measuring baseline GSH levels, whereas two other groups of oocytes were treated with $1 \mathrm{mM}$ hydrogen peroxide (Sigma). For the latter, one group was exposed to hydrogen peroxide for $90 \mathrm{~min}$ (oxidative insult), whereas the second group was incubated with hydrogen peroxide for $30 \mathrm{~min}$ and transferred to fresh culture medium without hydrogen peroxide for an additional $60 \mathrm{~min}$ (recovery after oxidative insult). During the last $15 \mathrm{~min}$ of 
incubation, monochlorobimane was added to a final concentration of $1 \mathrm{mM}$, after which the oocytes were washed in fresh culture medium. Imaging and micrograph analyses were then conducted using a deconvolution microscope equipped with a cyan fluorescent protein filter. The average fluorescence intensities were tabulated on a per oocyte basis, and the data were then compiled per group.

ROS. Formation of ROS was determined through the use of $2^{\prime}, 7^{\prime}$ dichlorodihydrofluorescein diacetate $\left(\mathrm{H}_{2} \mathrm{DCFDA}\right.$; Molecular Probes $)$ as described. ${ }^{28}$ The $\mathrm{H}_{2}$ DCFDA dye is membrane permeant, and upon entering the cell the acetate groups are hydrolyzed, creating a membrane impermeant form of the dye $\left(\mathrm{H}_{2} \mathrm{DCF}\right)$. Endogenous ROS oxidize this polar form of the dye to a quantifiable fluorogenic compound (DCF). Oocytes were mixed with a freshly prepared solution of $0.01 \mathrm{M} \mathrm{H}_{2}$ DCFDA and incubated for $15 \mathrm{~min}$. After extensive washing in fresh culture medium, imaging was carried out using a deconvolution microscope with an FITC filter. Total light intensity for each optical section and average light intensity for each oocyte were determined using the DeltaVision Software Analysis program. In order to determine baseline fluorescence, control oocytes (unstained) were incubated with an appropriate volume of vehicle (DMSO) before imaging. In some experiments, oocytes were cultured without or with pyruvate ( $10 \mathrm{mM}$; Sigma) for $6 \mathrm{~h}$ before being imaged for ROS content. In addition, the following inhibitors of the oxidative phosphorylation chain were used to assess specificity for mitochondrial ROS production: rotenone (inhibits Complex I activity), antimycin-A (inhibits Complex III activity) or oligomycin (inhibits Complex V activity). Each inhibitor $(2 \mu \mathrm{g} / \mathrm{ml})$ was individually added and the cultures were continued for 30 min before analysis of ROS content.

Somatic cell cultures. Ovarian somatic (granulosa) cells were isolated as described. ${ }^{29}$ Briefly, immature (21-24 days postpartum) FVB and B6C3F1 female mice were injected with $10 \mathrm{IU}$ of equine chorionic gonadotropin, and ovaries were removed $42 \mathrm{~h}$ later. The stimulated follicles were punctured with fine needles to collect granulosa cells into Waymouth's MB752/1 medium (Life Technologies) supplemented with penicillin, streptomycin and L-glutamine. After trypan blue staining, approximately $1 \times 10^{6}$ viable cells were seeded and cultured for $72 \mathrm{~h}$ in $100-\mathrm{mm}$ dishes containing $10 \mathrm{ml}$ of culture medium supplemented with $10 \%$ FBS (HyClone Laboratories, Logan, UT, USA). Mouse embryonic stem cells (line R1 derived from a 129 genetic background) were seeded at a density of approximately $2 \times 10^{6}$ cells per $0.1 \%$ gelatin-coated $100-\mathrm{mm}$ plate, cultured under standard conditions in a humidified incubator at $37^{\circ} \mathrm{C}$ under $5 \% \mathrm{CO}_{2}$ and passaged when the cultures reached $80 \%$ confluency.

Isolation of mitochondria. When the somatic cell cultures reached $80 \%$ confluency, $2 \mathrm{ml}$ of mitochondrial lysis buffer $(0.3 \mathrm{M}$ sucrose, $1 \mathrm{mM}$ EDTA, $5 \mathrm{mM}$ MOPS, $5 \mathrm{mM} \mathrm{KH}_{2} \mathrm{PO}_{4}, 0.1 \% \mathrm{BSA}$ ) were added to each plate, and the cells were removed using a cell scraper. The cell suspension was transferred into a small glass tissue bouncer and homogenized until smooth (approximately 10 up-and-down strokes), and the lysate was centrifuged at $600 \times g$ for $30 \mathrm{~min}$ at $4^{\circ} \mathrm{C}$. The supernatant was removed and spun at $10000 \times \mathrm{g}$ for $12 \mathrm{~min}$ at $4^{\circ} \mathrm{C}$, and the resulting crude mitochondrial pellet was resuspended in $0.2 \mathrm{ml}$ of $0.25 \mathrm{M}$ sucrose. This sample was then layered over a $25-60 \%$ Percoll density gradient diluted with $0.25 \mathrm{M}$ sucrose and centrifuged at $40000 \times \mathrm{g}$ for $20 \mathrm{~min}$ at $17^{\circ} \mathrm{C}$. The interface band was extracted from the gradient and washed in 2 volumes of $0.25 \mathrm{M}$ sucrose before a final centrifugation at $14000 \times g$ for $10 \mathrm{~min}$ at $4^{\circ} \mathrm{C}$ to yield a mitochondrial pellet, as described. ${ }^{30}$

Oocyte microinjection. Microinjection needles and holding pipettes were made using a Sutter puller (Sutter Instruments, Novato, CA, USA) and a De Fonbrune Microforge (EB Sciences, East Granby, CT, USA). The microinjection needles had inner diameters of $5 \mu \mathrm{m}$ with blunt tips. The experimental material to be injected or its negative control (mitochondria or sucrose, recombinant Rad51 or BSA, cytochrome $c$ or cytochrome $b$, recombinant Smac/DIABLO or BSA, respectively) was aspirated into the needle by negative suction. The mitochondrial suspension in sucrose (5-7 pl containing approximately $1 \times 10^{3}$ or $5 \times 10^{3}$ mitochondria from embryonic stem cells or granulosa cells, respectively), recombinant Rad51 $\left(6 \mathrm{pl}\right.$ of a $3.6 \mathrm{mg} / \mathrm{ml}$ stock per oocyte $\left.;{ }^{31}\right)$, cytochrome $c(6 \mathrm{pl}$ of a $400 \mu \mathrm{M}$ stock per oocyte) or recombinant Smac/DIABLO (6 pl of a $700 \mathrm{mg} / \mathrm{ml}$ stock: ${ }^{11}$ ) were injected into oocytes using a Piezo micromanipulator. Oocytes that did not survive the microinjection procedure (routinely less than $25 \%$ ) were discarded, and the remaining oocytes were transferred for culture and assessment of apoptosis.
Electron microscopy. Oocytes were cultured without or with pyruvate $(10 \mathrm{mM})$ for $2 \mathrm{~h}$ before being fixed for EM tomography. The oocytes were embedded in agarose and prepared for tomography using conventional protocols for good structural preservation. ${ }^{32}$ To survey the preservation quality of the oocytes, thinsectioned materials $(\sim 80 \mathrm{~nm})$ were examined using a JEOL 1200FX electron microscope. Three-dimensional reconstructions of portions of the cell containing mitochondria were generated using standard techniques. ${ }^{33}$ Sections with a thickness between 300 and $500 \mathrm{~nm}$ were cut out, and stained for $30 \mathrm{~min}$ in $2 \%$ aqueous uranyl acetate, followed by $30 \mathrm{~min}$ in lead salts. Next, fiducial cues consisting of $20 \mathrm{~nm}$ colloidal gold particles were deposited on both sides of each section.

For each reconstruction, a series of images at regular tilt increments were collected with a JEOL 4000EX intermediate-voltage electron microscope operated at $400 \mathrm{kV}$. In order to limit anisotropic specimen thinning during image collection, the specimens were irradiated before initiating a tilt series. Pre-irradiation in this manner subjected the specimen to the steepest portion of the nonlinear shrinkage profile before images were collected using a slow-scan CCD camera with $1960 \times 2560$ pixels at a resolution of $1.1 \mathrm{~nm}$. Tilt series were recorded at a magnification of $\times 20000$ with an angular increment of $2^{\circ}$ from $-60^{\circ}$ to $+60^{\circ}$ about an axis perpendicular to the optical axis of the microscope using a computer-controlled goniometer to achieve accurate increments at each angular step. Illumination was held to near parallel beam conditions and optical density maintained constant by varying the exposure time. The IMOD package ${ }^{34}$ was used for alignment and the TxBR package (National Center for Microscopy and Imaging Research, San Diego, CA, USA) was used for generating the reconstructions.

Volume segmentation was performed by manual tracing in the planes of highest resolution with the program Xvoxtrace. ${ }^{35,36}$ Mitochondrial reconstructions were visualized using Analyze (Mayo Foundation, Rochester, MN, USA) or Synu (National Center for Microscopy and Imaging Research) as described. ${ }^{37}$ These programs allow one to step through reconstructed slices in any orientation and to track or model features of interest in three dimensions. Measurements of structural features were made within segmented volumes by the programs, Synusurface and Synuvolume (National Center for Microscopy and Imaging Research). Quantitation of the presence of different mitochondrial structures was performed on random transmission EM-acquired images.

Cytochrome c release. Oocyte mitochondrial enriched fractions were prepared by differential centrifugation as described above. In some experiments, the oocytes were pre-incubated without or with pyruvate $(10 \mathrm{mM})$ for $2 \mathrm{~h}$. Cytochrome $c$ release was evaluated by a sensitive and specific immunoassay, using a commercial ELISA kit (Quantikine ${ }^{\mathrm{R}} \mathrm{M}$ Assay; R\&D Systems) ${ }^{38}$ according to the manufacturer's instructions. The light emitted was quantified by using a microtiter plate reader at $450 \mathrm{~nm}$, and translated into cytochrome $c$ concentrations through a standard curve.

Induction of calcium waves. After superovulation, FVB oocytes were subjected to treatment with ionomycin to mimic the $\mathrm{Ca}^{2+}$ oscillations that occur upon fertilization, as previously reported. ${ }^{39}$ Briefly, oocytes were treated with Acid Tyrode's Solution (Sigma Aldrich, Oakville, Ontario, Canada) to remove the zona pellucida. Oocytes were then washed several times and cultured in HTF with $0.5 \%$ BSA for $1 \mathrm{~h}$ to ensure complete removal of the acidic solution from the oocytes. At this point, the control group of oocytes was removed and cultured in HTF with $0.5 \%$ BSA for $6 \mathrm{~h}$ until the time of analysis. The remaining oocytes were placed in two dishes of Earle's balanced salt solution without $\mathrm{Ca}^{2+}$ (EBSS, Sigma, Oakville, Ontario, Canada). Ionomycin diluted in DMSO was added to a final concentration of $2 \mu \mathrm{M}$ and 14 min later, EBSS supplemented with $1.8 \mathrm{mM} \mathrm{Ca}^{2+}$ and ionomycin (final concentration of $2 \mu \mathrm{M}$ ) was added. Following $3 \mathrm{~min}$ in this culture environment, the oocytes were placed in a dish of EBSS with $10 \mu \mathrm{M}$ EGTA (Sigma, Oakville, Ontario, Canada), and cultured for $1 \mathrm{~h}$ before being washed and placed in HTF with $0.5 \%$ BSA until the time of analysis. Vehicle-treated oocytes were cultured in the same manner as ionomycin-treated oocytes, but were treated with DMSO lacking ionomycin.

Rad51 immunocytochemistry. Rad51 protein levels were assessed in oocytes, essentially as described previously. ${ }^{38,40}$ As mature metaphase II oocytes do not have a nucleus, immature (germinal vesicle-intact) oocytes were also studied to allow assessment of nuclear Rad51 levels. Briefly, immature and mature oocytes were harvested from young (7 weeks of age) C57BL/6 and AKR/J female mice, and fixed in neutral-buffered $10 \%$ formalin for at least $20 \mathrm{~min}$ at room temperature. After 
fixation, oocytes were washed twice in $1 \times$-phosphate-buffered saline (PBS) and stored at $4^{\circ} \mathrm{C}$ until the immunostaining procedure was performed. Permeabilization was achieved by a $10 \mathrm{~min}$ pre-exposure to $0.5 \%$ Triton-X in PBS followed by blocking in $1 \times$-PBS containing $10 \%$ goat serum and $0.05 \%$ Triton $X-100$. The oocytes were then immunostained overnight at $4^{\circ} \mathrm{C}$ using a $1: 35$ dilution of an affinity-purified mouse monoclonal antibody raised against full-length human Rad51 (Upstate USA, Inc., Charlottesville, VA, USA) prepared in $1 \times$-PBS supplemented with $1 \%$ goat serum. The slides were washed and incubated with a $1: 200$ dilution of an anti-mouse IgG conjugated with Oregon Green (Molecular probes). The slides were washed, counterstained with 4',6-diamidino-2-phenylindole (DAPI; Sigma) and subsequently viewed by deconvolution microscopy. Oocytes were serially scanned, and 10 optical sections were analyzed using DeltaVision Software. The total fluorescence was determined on stacked deconvolved images, and mean of relative fluorescent units (RFU) in each group was calculated for AKR/J or C57BL/6 oocytes. Oocytes without exposure to primary antibody were used as negative controls to determine background, which was subtracted from each value before final analysis.

Data presentation and statistical analysis. All experiments were independently replicated at least three times with different mice. Combined data from the replicate experiments were subjected to a one-way analysis of variance followed by Scheffe's $F$-test, Student's $t$-test or $\chi^{2}$ analysis, and $P$-values less than 0.05 were considered statistically significant. Graphs represent the mean $( \pm$ S.E.M.) of combined data from the replicate experiments, whereas representative photomicrographs of DNA damage (comet assay), EM-based analyses of mitochondria and Rad51 immunocytochemistry are presented.

Acknowledgements. We thank $X$ Wang for recombinant Smac/DIABLO, $M$ Gertsenstein for technical assistance, J Wrana and M Barrios-Rodiles for use of the luminometer, R Lawrence for assistance with the TxBR package and S Riley for assistance with figures. This work was supported by the National Institutes of Health (R01/R37-AG012279 to JLT; RR04050 to MHE), Vincent Memorial Research Funds (to JLT), the Harvard Center of Excellence on Women's Health (to GIP), Michigan State University (to GIP) and the Canadian Institutes of Health Research (MOP. 14058 to RFC and AJ; Doctoral Research Award to BMA).

1. Danial NN, Korsmeyer SJ. Cell death: critical control points. Cell 2004; 116: 205-219.

2. Li L, Zou L. Sensing, signaling, and responding to DNA damage: organization of the checkpoint pathways in mammalian cells. J Cell Biochem 2005; 94: 298-306.

3. Green DR, Kroemer G. The pathophysiology of mitochondrial cell death. Science 2004; 305: 626-629.

4. Jiang X, Wang X. Cytochrome c-mediated apoptosis. Annu Rev Biochem 2004; 73 87-106.

5. Thacker J. The RAD51 gene family, genetic instability and cancer. Cancer Lett 2005; 219 125-135.

6. Chipuk JE, Kuwana T, Bouchier-Hayes L, Droin NM, Newmeyer DD, Schuler M et al. Direct activation of Bax by p53 mediates mitochondrial membrane permeabilization and apoptosis. Science 2004; 303: 1010-1014.

7. Perez Gl, Knudson CM, Leykin L, Korsmeyer SJ, Tilly JL. Apoptosis-associated signaling pathways are required for chemotherapy-mediated female germ cell destruction. Nat Med 1997: 3: 1228-1332.

8. Perez GI, Tao XJ, Tilly JL. Fragmentation and death (a.k.a. apoptosis) of ovulated oocytes Mol Hum Reprod 1999; 5: 414-420.

9. Morita Y, Perez Gl, Paris F, Miranda SR, Ehleiter D, Haimovitz-Friedman A et al. Oocyte apoptosis is suppressed by disruption of the acid sphingomyelinase gene or by sphingosine-1-phosphate. Nat Med 2000; 6: 1109-1114.

10. Tilly JL. Commuting the death sentence: how oocytes strive to survive. Nat Rev Mol Cell Biol 2001; 2: 838-848.

11. Du C, Fang M, Li Y, Li L, Wang X. Smac, a mitochondrial protein that promotes cytochrome c-dependent caspase activation by eliminating IAP inhibition. Cell 2000; 102: 33-42.

12. Verhagen AM, Ekert PG, Pakusch M, Silke J, Connolly LM, Reid GE et al. Identification of DIABLO, a mammalian protein that promotes apoptosis by binding to and antagonizing IAP proteins. Cell 2000; 102: 43-53.
13. Okada H, Suh W-K, Jin J, Woo M, Du C, Elia A et al. Generation and characterization of Smac/DIABLO-deficient mice. Mol Cell Biol 2002; 22: 3509-3517.

14. Bridges CB. Specific modifiers of eosin eye color in Drosophila melanogaster. J Exp Zool 1919; 10: 337-384

15. Nadeau JH. Modifier genes and protective alleles in humans and mice. Curr Opin Genet Dev 2003; 13: 290-295.

16. Kuida K, Zheng TS, Na S, Kuan C, Yang D, Karasuyama $\mathrm{H}$ et al. Decreased apoptosis in the brain and premature lethality in CPP32-deficient mice. Nature 1996; 384: 368-372.

17. Leonard JR, Klocke BJ, D'Sa C, Flavell RA, Roth KA. Strain-dependent neurodevelopmental abnormalities in caspase-3-deficient mice. J Neuropathol Exp Neurol 2002; 61: 673-677.

18. Charames GS, Bapat B. Genomic instability and cancer. Curr Mol Med 2003; 3: 589-596

19. Lombard DB, Chua KF, Mostoslavsky R, Franco S, Gostissa M, Alt FW. DNA repair, genome stability, and aging. Cell 2005; 120: 497-512.

20. Schwarz ER, Pollick C, Dow J, Patterson M, Birnbaum Y, Kloner RA. A small animal model of non-ischemic cardiomyopathy and its evaluation by transthoracic echocardiography. Cardiovasc Res 1998; 39: 216-223.

21. Paumard P, Vaillier J, Coulary B, Schaeffer J, Soubannier V, Mueller DM et al. The ATP synthase is involved in generating mitochondrial cristae morphology. EMBO J 2002; 21: 221-230.

22. Lloyd D, Lemar KM, Salgado LE, Gould TM, Murray DB. Respiratory oscillations in yeast: mitochondrial reactive oxygen species, apoptosis and time; a hypothesis. FEMS Yeast Res 2003; 3: 333-339.

23. Jurisicova A, Lee HJ, D’Estaing SG, Tilly J, Perez GI. Molecular requirements for doxorubicin mediated death in murine oocytes. Cell Death Differ 2006; 13: 1466-1474.

24. Anderson D, Plewa MJ. The international comet assay workshop. Mutagenesis 1998; 13: 67-73.

25. Acton BM, Jurisicova A, Jurisica I, Casper RF. Alterations in mitochondrial membrane potential during preimplantation stages of mouse and human embryo development. $\mathrm{Mol}$ Hum Reprod 2004; 10: 23-32.

26. Bernas T, Dobrucki J. Mitochondrial and nonmitochondrial reduction of MTT: interaction of MTT with TMRE, JC-1, and NAO mitochondrial fluorescent probes. Cytometry 2002; 47: 236-242.

27. Nasr-Esfahani MH, Johnson MH. Quantitative analysis of cellular glutathione in early preimplantation mouse embryos developing in vivo and in vitro. Hum Reprod 1992; 7: 1281-1290.

28. Yang HW, Hwang KJ, Kwon HC, Kim HS, Choi KW, Oh KS. Detection of reactive oxygen species (ROS) and apoptosis in human fragmented embryos. Hum Reprod 1998; 13: $998-1002$.

29. Matikainen T, Perez Gl, Zheng TS, Kluzak TR, Rueda BR, Flavell RA et al. Caspase-3 gene knockout defines cell lineage specificity for programmed cell death signaling in the ovary. Endocrinology 2001; 142: 2468-2480.

30. Darley-Usmar VM, Rickwood D, Wilson MT. Isolation and characteristics of intact mitochondria In: Rickwood D, Hames BD (eds). Mitochondria, a Practical Approach. IRL Press: Oxford-Washington DC, 1987, pp 1-16

31. Kurumizaka H, Aihara H, Kagawa W, Shibata T, Yokoyama S. Human Rad51 amino acid residues required for Rad52 binding. J Mol Biol 1999; 291: 537-548.

32. Ricci JE, Munoz-Pinedo C, Fitzgerald P, Bailly-Maitre B, Perkins GA, Yadava N et al. Disruption of mitochondrial function during apoptosis is mediated by caspase cleavage of the p75 subunit of complex I of the electron transport chain. Cell 2004; 117: 773-786.

33. Perkins GA, Ellisman MH, Fox DA. Three-dimensional analysis of mouse rod and cone mitochondrial cristae architecture: bioenergetic and functional implications. Mol Vision 2003; 9: 60-73.

34. Mastronarde DN. Dual-axis tomography: an approach with alignment methods that preserve resolution. J Struct Biol 1997; 120: 343-352.

35. Perkins G, Renken C, Martone M, Young S, Ellisman MH, Frey T. Electron tomography of neuronal mitochondria: 3-D structure and organization of cristae and membrane contacts. J Struct Biol 1997; 119: 260-272.

36. Perkins G, Renken C, Song JY, Frey TG, Young S, Lamont S et al. Electron tomography of large, multicomponent biological structures. J Struct Biol 1997; 120: 219-227.

37. Perkins GA, Renken CW, Frey TG, Ellisman MH. Membrane architecture of mitochondria in neurons of the central nervous system. J Neurosci Res 2001; 66: 857-865.

38. Perez Gl, Jurisicova A, Matikainen T, Moriyama T, Kim MR, Takai Y et al. A central role for ceramide in the age-related acceleration of apoptosis in the female germline. FASEB $J$ 2005; 19: 860-862

39. Markoulaki S, Matson S, Abbott AL, Ducibella T. Oscillatory CaMKIl activity in mouse egg activation. Dev Biol 2003; 258: 464-474.

40. Matikainen T, Perez GI, Jurisicova A, Pru JK, Schlezinger JJ, Ryu HY et al. Aromatic hydrocarbon receptor-driven Bax gene expression is required for premature ovarian failure caused by biohazardous environmental chemicals. Nat Genet 2001; 28: 355-360.

\section{Supplementary Information accompanies the paper on Cell Death and Differentiation website (http://www.nature.com/cdd)}

